\title{
ON TRANSFORMATIONS OF DERIVATIVES
}

\section{A. M. BRUCKNER ${ }^{1}$}

ABSTRACT. Let $f$ be a derivative on $[a, b], \phi$ a continuous function on the real line and $h$ a homeomorphism of $[a, b]$ onto itself. We study the problem of determining conditions under which $\phi \circ f$ or $f \circ h$ are derivatives.

1. Introduction and preliminaries. The property of being a derivative is not, in general, preserved under compositions (on the inside or outside) with continuous functions. Thus, Maximoff $[5]$ has shown that if $f$ is any Darboux function in the first class of Baire on $[0,1]$, there exists a homeomorphism $h$ of $[0,1]$ onto itself such that $g=f \circ h$ is a derivative. It follows that while $g$ is a derivative, $g \circ b^{-1}=f$ need not be a derivative. Furthermore, Choquet [2] has shown that if $\phi$ is a continuous function, not linear, there exists a bounded derivative $f$ such that $\phi \circ f$ is not a derivative. The main purpose of this article is to give conditions on derivatives $f$, continuous functions $\phi$ and homeomorphisms $h$ which guarantee that $\phi \circ f$ and $f \circ h$ are derivatives. We shall see that the class of functions which, along with their squares, are derivatives, play an important role in our consideration. In the sequel, we shall be concerned with real-valued functions defined on an interval $[a, b]$. When we say a function $f$ is a derivative we mean that there exists a function $F$ such that $F^{\prime}(x)=f(x)$ for all $x$ in $[a, b]$. We shall denote Lebesgue measure by the letter $\lambda$.

2. A theorem on functions which together with the ir squares are derivatives. Theorem 1 below will be useful in $\$ \$ 3$ and 4 .

Theorem 1. If $f$ and $f^{2}$ are derivatives on $[a, b]$, then every point of $[a, b]$ is a Lebesgue point for $f$.

Proof. We first note that $f^{2}$ is summable because it is the derivative of

Received by the editors December 17, 1973.

AMS (MOS) subject classifications (1970). Primary 26A24; Secondary 26A36.

Key words and phrases. Derivative, Lebesgue point, approximate continuity, approximate derivative.

1 The author was supported in part by NSF grant GP 18968. 
an increasing function. Therefore $f$ is a lso summable. It is clear that both $f$ and $f^{2}$ are the derivatives of their integrals.

We now show that $f$ is approximately continuous. Let $x_{0} \in[a, b]$. Since the functions $f-f\left(x_{0}\right)$ and $\left(f-f\left(x_{0}\right)\right)^{2} \equiv f^{2}-2 f f\left(x_{0}\right)+f^{2}\left(x_{0}\right)$ are derivatives, we may assume that $f\left(x_{0}\right)=f^{2}\left(x_{0}\right)=0$. Let $\epsilon>0$, and let $A=\{x:|f(x)| \geq \epsilon\}$. We wish to show that $x$ is a point of dispersion of $A$. Let

$$
I^{h}=\frac{1}{b} \int_{x_{0}}^{x_{0}+h} f^{2} d \lambda \text {, and } I_{A}^{h}=\frac{1}{|h|} \int_{\left[x_{0}, x_{0}+h\right] \cap A} f^{2} d \lambda .
$$

Since $f^{2}$ is the derivative of its integral, $\lim _{h \rightarrow 0} I^{h}=f^{2}\left(x_{0}\right)=0$. Therefore $\lim _{h \rightarrow 0} I_{A}^{h}=0$. On the other hand

$$
I_{A}^{h} \geq \epsilon^{2} \lambda\left(A \cap\left[x_{0}, x_{0}+b\right]\right) /|h| .
$$

Thus $\lim _{h \rightarrow 0} \lambda\left(A \cap\left[x_{0}, x_{0}+b\right]\right) /|b|=0$. Since $\epsilon$ was arbitrary, $x_{0}$ is a point of dispersion of $A$, and $f$ is approximately continuous at $x_{0}$.

To show that each point of $[a, b]$ is a Lebesgue point for $f$, we again let $x_{0} \in[a, b], \epsilon>0$ and assume $f\left(x_{0}\right)=0$. We wish to show

$$
\lim _{h \rightarrow 0} \frac{1}{|h|} \int_{x_{0}}^{x_{0}+h}|f| d \lambda=0
$$

Write

$$
\begin{aligned}
\frac{1}{|b|} \int_{x_{0}}^{x_{0}+h}|f| d \lambda & \\
= & \frac{1}{|b|} \int_{\left[x_{0}, x_{0}+h\right] \cap A_{1}}|f| d \lambda+\frac{1}{|h|} \int_{\left[x_{0}, x_{0}+h\right] \cap A_{2}}|f| d \lambda \\
& +\frac{1}{|h|} \int_{\left[x_{0}, x_{0}+h\right] \cap A_{3}}|f| d \lambda
\end{aligned}
$$

where $A_{1}=\{x:|f(x)|<\epsilon\}, A_{2}=\{x: \epsilon \leq|f(x)|<1\}$ and $A_{3}=\{x:|f(x)| \geq 1\}$. Then

$$
\begin{aligned}
& \frac{1}{|b|} \int_{\left[x_{0}, x_{0}+h\right] \cap A_{1}}|f| d \lambda \leq \epsilon \frac{\lambda\left(\left[x_{0}, x_{0}+b\right] \cap A_{1}\right)}{|b|} \leq \epsilon, \\
& \frac{1}{|b|} \int_{\left[x_{0}, x_{0}+h\right] \cap A_{2}}|f| d \lambda \leq \frac{1}{|b|} \lambda\left(\left[x_{0}, x_{0}+h\right] \cap A_{2}\right)<\epsilon
\end{aligned}
$$


for sufficiently small $|h|$ because $f$ is approximately continuous at $x_{0}$, and

$$
\frac{1}{|b|} \int_{\left[x_{0}, x_{0}+h\right] \cap A_{3}}|f| d \lambda \leq \frac{1}{|h|} \int_{\left[x_{0}, x_{0}+h\right] \cap A_{3}} f^{2} d \lambda \leq \frac{1}{|h|} \int_{x_{0}}^{x_{0}+h} f^{2} d \lambda<\epsilon
$$

for sufficiently small $|h|$ because $f^{2}$ is the derivative of its integral at $x_{0}$ and $f^{2}\left(x_{0}\right)=0$. It follows from these inequalities that $|h|^{-1} \int_{x_{0}}^{x_{0}+h}|f| d \lambda<$ $3 \epsilon$ for sufficiently small $|h|$. Since $\epsilon$ was arbitrary,

$$
\lim _{h \rightarrow 0} \frac{1}{h} \int_{x_{0}}^{x_{0}+h}|f| d \lambda=0
$$

and $x$ is a Lebesgue point for $f$. This completes the proof of Theorem 1.

3. On the composition $\phi \circ h$. Suppose now that $f$ is bounded and $f$ and $f^{2}$ are derivatives. Then $f$ is approximately continuous by Theorem 1 . If $\phi$ is any continuous function defined on the real line $R$, then $\phi \circ f$ is a bounded approximately continuous function and is therefore a derivative. (This result was established with a different proof by Wilcosz [6].) Example 1 below shows that one cannot obtain the corresponding result if one drops the requirement that $f$ be bounded. Nonetheless, The orem 2 below shows that one can obtain the result for certain classes of continuous functions $\phi$.

Example 1. Let $I_{n}=\left[10^{-2 n}, 10^{-2 n}+10^{-4 n}\right]$. Define a function $g$ as follows: $g(x)=10^{n}$ on $I_{n}, g(x)=0$ elsewhere on $[0,1]$. Let $G(x)=\int_{0}^{x} g d \lambda$ and $H(x)=\int_{0}^{x} \sqrt{ } g d \lambda$. It is easy to verify that $G^{\prime}(0)=H^{\prime}(0)=0$, but that $\int_{I_{n}} g^{4} d \lambda=1$ so that the function $g^{4}$ is not summable on $[0,1]$. Now replace $\sqrt{ } g$ by a function $f$ which equals $\sqrt{ } g$ on $I_{n}$ but which, on the intervals $\left[10^{-2 n}-10^{-4 n}, 10^{-2 n}\right]$ and $\left[10^{-2 n}+10^{-4 n}, 10^{-2 n}+2 \cdot 10^{-4 n}\right]$, is linear and vanishes elsewhere in $[0,1]$ so that $f$ is continuous on $[0,1]$. Then $f$ and $f^{2}$ are the derivatives of their integrals everywhere on $[0,1]$, but $f^{8}=g^{4}$ is not summable on $[0,1]$. Since $f^{8} \geq 0$, it cannot be a derivative because a nonnegative derivative is summable. This example shows that Theorem 2 below does not apply to the function $\phi(x)=x^{8}$.

Theorem 2. Let $f, f^{2}$ be derivatives on $[a, b]$, and let $\phi$ be continuous on $R$. Then:

(a) If $\phi$ is bounded or satisfies a Lipschitz condition, the function $\phi \circ f$ has each point as a Lebesgue point. In particular, $\phi \circ f$ is a derivative. 
(b) If $\phi \circ f-f$ or $\phi \circ f-f^{2}$ is bounded, $\phi \circ f$ is a derivative.

Proof. (a) If $\phi$ is bounded, then $\phi \circ f$ is a bounded approximately continuous function and thus each point is a Lebesgue point. If $\phi$ satisfies the Lipschitz condition $|\phi(y)-\phi(x)| \leq M|y-x|$ for all real $x$ and $y$, then

$$
\frac{1}{|b|} \int_{x_{0}}^{x_{0}+h}\left|\phi \circ f(x)-\phi \circ f\left(x_{0}\right)\right| d x \leq \frac{M}{|b|} \int_{x_{0}}^{x_{0}+h}\left|f(x)-f\left(x_{0}\right)\right| d x,
$$

and this last expression approaches 0 with $h$ because each point $x_{0}$ is a Lebesgue point for $f$ by Theorem 1 .

(b) Let $g=\phi \circ f-f$. Now $f$ is approximately continuous by Theorem 1 . Thus $\phi \circ f$ is also approximately continuous so $g$ is approximately continuous. If $g$ is also bounded, $g$ is a derivative. Thus $\phi \circ f=f+g$ is also a derivative. A similar argument shows $\phi \circ f$ is a derivative if $\phi \circ f-f^{2}$ is bounded.

We now show that Theorems 1 and 2 remain valid if we assume only that $f$ and $f^{2}$ are approximate derivatives.

Theorem 3. If $f$ and $f^{2}$ are approximate derivatives, then $f$ and $f^{2}$ are derivatives.

Proof. Since any approximate derivative which dominates a derivative is itself a derivative [3], we note that the function $f^{2} \geq 0$ is a derivative. Since $f \geq-\left(f^{2}+1\right) / 2, f$ is also a derivative.

4. On the composition $f \circ h$. Maximoff's the orem [5] implies that a homeomorphic change of variables may destroy the property of being a derivative. Example 2 below shows that even if $f$ is a bounded derivative and $h$ and $b^{-1}$ satisfy a Lipschitz condition, the function $f \circ h$ might fail to be a derivative. On the other hand, if $f$ and $f^{2}$ are derivatives, and $b$ and $b^{-1}$ satisfy a Lipschitz condition then, according to The orem 4, below, $f \circ h$ and $(f \circ h)^{2}$ are derivatives. Thus the class of functions which together $w$ ith the ir squares are derivatives is closed under this type of change of variables.

Example 2. Let $\left\{a_{n}\right\},\left\{b_{n}\right\},\left\{c_{n}\right\}$ and $\left\{d_{n}\right\}$ be decreasing sequences of positive numbers such that:

(1) $d_{1}=1$,

(2) $d_{n}>c_{n}>b_{n}>a_{n}>d_{n+1}$ for all $n$,

(3) $\lim _{n \rightarrow \infty} d_{n}=0$,

(4) $\bigcup_{n=1}^{\infty}\left[a_{n}, b_{n}\right]$ has density $1 / 2$ at the origin, 
(5) $\bigcup_{n=1}^{\infty}\left[b_{n}, c_{n}\right]$ has density 0 at the origin,

(6) $\bigcup_{n=1}^{\infty}\left[c_{n}, d_{n}\right]$ has density $1 / 2$ at the origin, and

(7) $\bigcup_{n=2}^{\infty}\left[d_{n}, a_{n-1}\right]$ has density 0 at the origin.

Let $f$ be the function satisfying

$$
f(0)=0, \quad f(x)=-1 \text { on } \bigcup_{n=1}^{\infty}\left[a_{n}, b_{n}\right], \quad f(x)=1 \text { on } \bigcup_{n=1}^{\infty}\left[c_{n}, d_{n}\right],
$$

and $f$ is linear on $\left[b_{n}, c_{n}\right]$ and $\left[d_{n}, a_{n-1}\right]$ in such a way that $f$ is continuous everywhere except at $x=0$. It is easy to verify that $f$ is the derivative of its integral: this follows at $x=0$ by a direct computation and it follows elsewhere from the continuity of $f$.

Now let $\left\{a_{n}^{\prime}\right\},\left\{b_{n}^{\prime}\right\},\left\{c_{n}^{\prime}\right\}$ and $\left\{d_{n}^{\prime}\right\}$ be sequences of positive numbers such that for all $n$ :
(1) $a_{n}^{\prime}=a_{n}$,
(2) $d_{n}^{\prime}=d_{n}$,
(3) $d_{n}^{\prime}>c_{n}^{\prime}>b_{n}^{\prime}>a_{n}^{\prime}$,
(4) $b_{n}^{\prime}-a_{n}^{\prime}=1 / 2\left(b_{n}-a_{n}\right)$, and
(5) $d_{n}^{\prime}-c_{n}^{\prime}=3\left(d_{n}-c_{n}\right) / 2$.

Let $h$ be a homeomorphism of $[0,1]$ onto itself such that for each $n, h$ maps $\left[a_{n}, a_{n-1}\right]$ onto itself with $h(0)=0, b\left(a_{n}\right)=a_{n}^{\prime}, b\left(b_{n}\right)=b_{n}^{\prime}, b\left(c_{n}\right)=c_{n}^{\prime}$, $b\left(d_{n}\right)=d_{n}^{\prime}$, and $h$ linear on each of the intervals $\left[a_{n}, b_{n}\right],\left[b_{n}, c_{n}\right],\left[c_{n}, d_{n}\right]$, and $\left[d_{n}, a_{n-1}\right]$. It is easy to verify that $b^{-1}$ satisfies the Lipschitz condition

$$
\frac{1}{2}|y-x| \leq\left|h^{-1}(y)-b^{-1}(x)\right| \leq \frac{3}{2}|y-x| \text { for all } x \text { and } y,
$$

yet the number $1 / 2$ is a derived number of the integral of $f \circ b^{-1}$ at the origin while $f \circ h^{-1}(0)=0$. If $f \circ h^{-1}$ were a derivative, it would have to be the derivative of its integral. Therefore $f \circ h^{-1}$ is not a derivative.

Theorem 4. If $f$ and $f^{2}$ are derivatives on $[a, b]$ and $h$ is a homeomorphism of $[a, b]$ onto itself such that $h$ and $b^{-1}$ satisfy a Lipschitz condition, then $f \circ h$ and $(f \circ h)^{2}$ are derivatives.

Proof. Let $t_{0} \in[a, b]$. Write $x=h(t)$ and $x_{0}=h\left(t_{0}\right)$ and assume $h$ is an increasing homeomorphism such that $M^{-1}\left|t-t_{0}\right| \leq\left|h(t)-b\left(t_{0}\right)\right| \leq M\left|t-t_{0}\right|$ for some positive number $M$, and all $t$ in $[a, b]$. We first show that $t_{0}$ is a Lebesgue point for $f \circ h$. Let $t_{1}$ be a point in $[a, b]$ different from $t_{0}$ and let $x_{1}=h\left(t_{1}\right)$. Then 


$$
\begin{aligned}
&\left|\frac{1}{t_{1}-t_{0}} \int_{t_{0}}^{t} 1 f(h(t))-f\left(b\left(t_{0}\right)\right)\right| d t \mid \\
&=\left|\frac{1}{b^{-1}\left(x_{1}\right)-b^{-1}\left(x_{0}\right)} \int_{x_{0}}^{x_{1}}\right| f(x)-f\left(x_{0}\right)\left|h^{\prime-1}(x) d x\right| \\
& \leq \frac{M^{2}}{\left|x_{1}-x_{0}\right|} \int_{x_{0}}^{x_{1}}\left|f(x)-f\left(x_{0}\right)\right| d x,
\end{aligned}
$$

and this last expression a pproaches 0 as $x_{1} \rightarrow x_{0}$ because every point is a Lebesgue point for $f$.

We now invoke a theorem of Ios ifescu [4] according to which we need only show that

$$
\lim _{t_{1} \rightarrow t_{0}} \frac{1}{t_{1}-t_{0}} \int_{t_{0}}^{t}\left|f(h(t))-f\left(b\left(t_{0}\right)\right)\right|^{2} d t=0
$$

in order to guarantee that $(f \circ h)^{2}$ is also a derivative. But, as above, we see that

$$
\left|\frac{1}{t_{1}-t_{0}} \int_{t_{0}}^{t_{1}}\right| f(h(t))-\left.f\left(h\left(t_{0}\right)\right)\right|^{2} d t|\leq| \frac{M^{2}}{x_{1}-x_{0}} \int_{x_{0}}^{x_{1}}\left|f(x)-f\left(x_{0}\right)\right|^{2} d x \mid,
$$

and this last expression approaches 0 as $x_{1} \rightarrow x_{0}$ because $f$ and $f^{2}$ are derivatives [4].

Remark. We note from the proof of Theorem 4 that if every point of $[a, b]$ is a Lebesgue point for $f$, and $h$ is as in Theorem 4, then every point of $[a, b]$ is a Lebesgue point of $f \circ h$. We also note [1] that such homeomorphisms preserve approximately continuous functions: if $f$ is approximately continuous and $h$ is as above, then $f \circ b$ is approximately continuous. Yet, Example 2 shows that the property of being a bounded derivative is not preserved under such a change of variable. We also note that the Lipschitz requirements on $h$ cannot be dropped in the statement of Theorem 4. In fact, we have

Theorem 5. Let $f$ be a derivative on $[0,1]$. A necessary and sufficient condition for $f \circ h$ to be a derivative for every homeomorphism $h$ of $[0,1]$ onto itself is that $f$ be continuous.

Proof. The sufficiency is obvious. 
To prove necessity of the condition we assume $f$ is discontinuous at $x_{0}$ and consider the case that $x_{0}=0$, the proof being similar if $0<x_{0} \leq 1$. Since every derivative is a Darboux function, the cluster set of $f$ at 0 contains a point $y_{0}$, different from $f(0)$. Let $g(x)=f(x)$ if $x \neq 0, g(0)=y_{0}$. Since $g$ is a Darboux Baire 1 function, there exists by Maximoff's theorem a homeomorphism $h$ of $[0,1]$ onto itself such that $g \circ h$ is a derivative. But then $g \circ h(x)=f \circ h(x)$ for all $x$ except $x=0$, at which point we have $g \circ$ $h(0)=y_{0}$ and $f \circ h(0)=f(0)$. But if two functions agree everywhere except at one point, at least one of them must fail to be a derivative. Since $g \circ h$ is a derivative, $f \circ h$ is not.

\section{REFERENCES}

1. A. M. Bruckner, Density preserving homeomorphisms and a theorem of Maximoff, Quart. J. Math. Oxford Ser. (2) 21 (1970), 337-347. MR 42 \#3232.

2. G. Choquet, Application des propriétés descriptives de la fonction contingent à la théorie des fonctions de variable réelle et à la géométrie differentielle des variétés cartisiennes, J. Math. Pures Appl. (9) 26 (1947), 115-226 (1948). MR 9, 419.

3. C. Goffman and C. Neugebauer, On approximate derivatives, Proc. Amer. Math. Soc. 11 (1960), 962-966. MR 22 \#9562.

4. I. Iosifescu, Conditions that the product of two derivatives be a derivative, Rev. Roumaine Math. Pures Appl. 4 (1959), 641-649. (Russian) MR 22 \#11083.

5. I. Maximoff, On continuous transformation of some functions into an ordinary derivative, Ann. Scuola Norm. Sup. Pisa (2) 12 (1943), 147-160 (1947). MR 9, 419. $145-154$.

6. W. Wilcosz, Some properties of derivative functions, Fund. Math. 2 (1921),

DEPARTMENT OF MATHEMATICS, UNIVERSITY OF CALIFORNIA, SANTA BARBARA, CALIF ORNIA 93106 